\title{
A CLINICAL STUDY OF 100 CASES OF ECLAMPSIA AND 50 CASES OF SEVERE PREECLAMPSIA WITH OUTCOME OF TREATMENT WITH MAGNESIUM SULPHATE AND KEEPING LYTIC COCKTAIL AS CONTROL IN 10 CASES OF ECLAMPSIA
}

\author{
Shilpi Rani ${ }^{1}$, Bratati Moitra ${ }^{2}$
}

${ }_{1}^{1}$ DD, Department of Obstetrics and Gynaecology, Rajendra Institute of Medical Sciences, Ranchi, Jharkhand, India.

${ }^{2}$ Associate Professor, Department of Obstetrics and Gynaecology, Rajendra Institute of Medical Sciences, Ranchi, Jharkhand, India.

\section{BACKGROUND}

ABSTRACT

Eclampsia and severe preeclampsia are still major contributors to maternal mortality all over the world. Although, incidence has gone down in developed countries, incidence is still very high in developing countries. In our country, incidence of preeclampsia is $5-15 \%$, whereas incidence of eclampsia among hospital admissions is 1 in 500 to 1 in 30 . Incidence varies among different areas of same country. Lack of awareness for antenatal check-up, illiteracy, unavailability of quality antenatal check-up facility etc. are main causes why PIH and Eclampsia are diagnosed late and many a times patients come with complications like pulmonary oedema, coagulation failure, accidental haemorrhage, acute renal failure etc. Gold standard treatment of severe preeclampsia and eclampsia is $\mathrm{MgSO}_{4}$ along with antihypertensive and induction of labour to expedite the delivery within $12 \mathrm{hrs}$. and $24 \mathrm{hrs}$. in eclampsia and severe preeclampsia respectively. But still in remote areas, $\mathrm{MgSO}_{4}$ is not available.

This study was undertaken to see the outcome of treatment with $\mathrm{MgSO}_{4}$ in 100 cases of Eclampsia and 50 cases of severe Preeclampsia, keeping lytic Cocktail (Menon 1961, chlorpromazine, promethazine and pethidine injections) as control in 10 cases of Eclampsia.

\section{KEY WORDS}

Preeclampsia, Eclampsia, $\mathrm{MgSO}_{4}$, Lytic Cocktail.

HOW TO CITE THIS ARTICLE: Rani S, Moitra B. A clinical study of 100 cases of eclampsia and 50 cases of severe preeclampsia with outcome of treatment with magnesium sulphate and keeping lytic cocktail as control in 10 cases of eclampsia. J. Evolution Med. Dent. Sci. 2018;7(28):3248-3252, DOI: 10.14260/jemds/2018/730

\section{BACKGROUND}

Preeclampsia is a condition where BP is more than $140 / 90$ $\mathrm{mmHg}$ with proteinuria with or without oedema after 20 weeks of pregnancy. When PET is associated with convulsion, it is eclampsia (other causes of convulsion being ruled out).

Eclampsia is one of the dreaded complications of pregnancy. Incidence of eclampsia has gone down in developed countries from $0.7 \%$ (McDonald, 1976) to $0.05 \%$ (Alexander et al, 2004).

In India too incidence has declined, but still remains very high from $0.1 \%$ to $2 \%$ (Ratnam, Vol. 11, 1994). M. Chandra and Bhardwaj (1998) found that incidence of eclampsia was $1.56 \%$. Situation is gloomy in this part of the country.

Here, incidence of eclampsia is 2.7 to $4 \%$ and maternal mortality due to this is $27-30 \%$. Perinatal mortality is particularly high following eclampsia (Collab trial 1995; Douglas 1994).[1]

Illiteracy, poverty, lack of awareness, lack of facility for quality antenatal check-up in remote areas, poor transport facility and road conditions all together are responsible for high incidence of eclampsia and maternal mortality.

\section{MATERIALS AND METHODS}

100 cases of eclampsia and 50 cases of severe preeclampsia who were admitted in labour room of Rajendra Institute of Medical Sciences, Ranchi between September 2005 and

'Financial or Other Competing Interest': None.

Submission 12-07-2017, Peer Review 04-05-2018,

Acceptance 12-05-2018, Published 09-07-2018.

Corresponding Author:

Dr. Bratati Moitra,

\#24, Bardhaman Compound,

P. O. Lalpur, Ranchi, Jharkhand.

E-mail: bgs2104@yahoo.co.in

DOI: $10.14260 /$ jemds $/ 2018 / 730$
September 2006 were studied. Of these 100 cases of eclampsia, 90 cases were given $\mathrm{MgSO}_{4}$ as anticonvulsant and in 10 cases lytic cocktail was used.

- Common regimen of magnesium sulphate used was loading dose of $4 \mathrm{gm}$ of $20 \%$ magnesium sulphate solution given IV slowly +10 gms of $50 \%$ magnesium sulphate given IM $5 \mathrm{gm}$ in each buttock. Maintenance dose of $5 \mathrm{gm}$ of $50 \%$ magnesium sulphate IM was repeated 4 hourly till $24 \mathrm{hrs}$. after delivery or convulsion whichever is later (Dinsdale 1988; Pritchard 1955; Zuspan 1978).[2,3,4] Lytic cocktail is a mixture of drugs used for women with eclampsia. These are usually chlorpromazine, promethazine and pethidine (meperidine). First introduced in India by (Menon 1961).[5] These combination of drugs was thought to lower blood pressure and sedate the central nervous system.

- The mode of action for magnesium sulphate in control of eclamptic seizures and prevention of recurrent convulsions is still not clearly understood. This anticonvulsant activity may be mediated by magnesium's role as an N-Methyl-D-Aspartate (NMDA) antagonist (Euser 2009).[6]

- Magnesium may prevent and control eclamptic seizures by inhibiting NMDA receptors. Other possible mechanism are that magnesium sulphate may lead to cerebral vasodilatation with subsequent reduction of cerebral ischaemia (Belfort 1992).[7]

Among the 50 cases of severe preeclampsia, in 10 cases $\mathrm{MgSO}_{4}$ was not given and was kept as control. Soon after arrival of patient, a quick history was taken from the attendant and in the meantime, treatment was also started simultaneously. Patients were divided into different groups according to age and parity of patient. 
Quick Enquiry was done about-

Patient is booked or unbooked case

- No. of antenatal check-ups done.

- Presenting complaint was noted.

\section{Mode of Onset}

Any premonitory symptom was present or not, e.g. severe headache, blurred vision, epigastric pain, vomiting, diminished vision etc.-

- Time of onset of $1^{\text {st }}$ convulsion.

- Number of convulsions thereafter.

- Any symptom of labour pain.

- In case of post-partum eclampsia- interval between delivery and onset of fit was noted.

- Details of treatment received outside before coming to hospital.

\section{Menstrual History}

Gestational Age was Calculated, and Patients were divided in Three Groups-

- Less than 34 weeks.

- Between 34 - 37 weeks.

- $\quad$ Between 37 - 40 weeks.

\section{Obstetrical History}

Patients were divided in Five Groups of having Parity:

- Zero.

- One.

- Two.

- Three.

- Four or more.

\section{Past History}

\section{Detailed Relevant Past History}

- Seizure disorder.

- Past history such as diabetes, chronic hypertension and autoimmune disease (Duckitt 2005).[8]

- Renal disorder.

- Toxaemia of pregnancy in previous confinement if any.

Family History

- Hypertension

- Diabetes.

- Toxaemia of pregnancy in $1^{\text {st }}$ degree relative.

- Thorough general examination.

- Obstetrical examination.

\section{Important Findings of the Study}

- The general incidence of severe preeclampsia was $3.45 \%$, i.e. 1 in 29 .

- $\quad$ The general incidence of eclampsia was $5.03 \%$, i.e. 1 in 20.

- Maximum incidence was found in patients of less than 20 years of age.

- Most patients belonged to low socioeconomic status with $82.7 \%$ of patients having no antenatal check-ups.

- $71.4 \%$ of patients were primigravida in both eclampsia and severe preeclampsia.

- Antepartum eclampsia being the commonest $77 \%$ with intrapartum eclampsia $8 \%$ and postpartum eclampsia $15 \%$.
- $55 \%$ patients were admitted within 12 hours of convulsive attack.

\begin{tabular}{|c|c|c|c|}
\hline $\begin{array}{c}\text { Treatment } \\
\text { Regimen }\end{array}$ & $\begin{array}{c}\text { Antepartum } \\
\text { Eclampsia }\end{array}$ & $\begin{array}{c}\text { Intrapartum } \\
\text { Eclampsia }\end{array}$ & $\begin{array}{c}\text { Postpartum } \\
\text { Eclampsia }\end{array}$ \\
\hline $\begin{array}{c}\text { Magnesium } \\
\text { Sulphate }\end{array}$ & 73 & 6 & 11 \\
\hline Lytic Cocktail & 4 & 2 & 4 \\
\hline Total & 77 & 8 & 15 \\
\hline Percentage & $77 \%$ & $8 \%$ & $15 \%$ \\
\hline \multicolumn{4}{|c|}{ Table 1. Distribution of Cases according to Types of } \\
Eclampsia \\
\hline
\end{tabular}

\begin{tabular}{|c|c|c|c|c|}
\hline $\begin{array}{c}\text { Systolic } \\
\text { Blood } \\
\text { Pressure } \\
\text { (mmHg) }\end{array}$ & $\begin{array}{c}\text { Severe Preeclampsia } \\
\text { Sulphate } \\
\text { Group }\end{array}$ & $\begin{array}{c}\text { Control } \\
\text { Group }\end{array}$ & $\begin{array}{c}\text { Magnesium } \\
\text { Sulphate } \\
\text { Group }\end{array}$ & $\begin{array}{c}\text { Lytic } \\
\text { Cocktail } \\
\text { Group }\end{array}$ \\
\hline $140-158$ & 0 & 0 & 7 & 2 \\
\hline $160-200$ & 33 & 7 & 63 & 6 \\
\hline$>200$ & 7 & 3 & 20 & 2 \\
\hline \multicolumn{5}{|c|}{ Diastolic Blood Pressure (mmHg) } \\
\hline $90-108$ & 0 & 0 & 12 & 3 \\
\hline $110-120$ & 28 & 6 & 61 & 5 \\
\hline$>120$ & 12 & 4 & 17 & 2 \\
\hline
\end{tabular}

Table 2. Systolic and Diastolic Blood Pressure at the Time of Admission

$\mathrm{p}=5.991$

\begin{tabular}{|c|c|c|c|c|c|c|c|c|}
\hline \multirow{3}{*}{$\begin{array}{l}\text { Diastolic } \\
\text { Blood } \\
\text { Pressure } \\
\text { (mmHg) }\end{array}$} & \multicolumn{4}{|c|}{ Severe Preeclampsia } & \multicolumn{4}{|c|}{ Eclampsia } \\
\hline & \multicolumn{2}{|c|}{$\begin{array}{c}\text { Magnesium } \\
\text { Sulphate } \\
\text { Group }\end{array}$} & \multicolumn{2}{|c|}{$\begin{array}{c}\text { Control } \\
\text { Group }\end{array}$} & \multicolumn{2}{|c|}{$\begin{array}{l}\text { Magnesium } \\
\text { Sulphate } \\
\text { Group }\end{array}$} & \multicolumn{2}{|c|}{$\begin{array}{c}\text { Lytic } \\
\text { Cocktail } \\
\text { Group }\end{array}$} \\
\hline & $\begin{array}{c}\text { Pre } \\
\text { Rx }\end{array}$ & $\begin{array}{c}\text { Post } \\
\text { Rx }\end{array}$ & $\begin{array}{c}\text { Pre } \\
\text { Rx }\end{array}$ & $\begin{array}{c}\text { Post } \\
\text { Rx }\end{array}$ & $\begin{array}{c}\text { Pre } \\
\text { Rx }\end{array}$ & $\begin{array}{c}\text { Post } \\
\text { Rx }\end{array}$ & $\begin{array}{c}\text { Pre } \\
\text { Rx }\end{array}$ & $\begin{array}{c}\text { Post } \\
\text { Rx }\end{array}$ \\
\hline 90 to 108 & 0 & 24 & 0 & 4 & 2 & 55 & 1 & 4 \\
\hline$>1$ & 40 & 16 & 10 & 6 & 88 & 33 & 9 & 5 \\
\hline & & & & & \multicolumn{2}{|c|}{$\begin{array}{l}2 \text { patients } \\
\text { died within } \\
24 \text { hours of } \\
\text { treatment }\end{array}$} & \multicolumn{2}{|c|}{$\begin{array}{l}1 \text { patient } \\
\text { died within } \\
24 \text { hours of } \\
\text { treatment }\end{array}$} \\
\hline & & & & & & & & \\
\hline
\end{tabular}

$\mathrm{p}=3.84$

\begin{tabular}{|c|c|c|c|c|}
\hline & \multicolumn{2}{|c|}{$\begin{array}{c}\text { Severe } \\
\text { Preeclampsia }\end{array}$} & \multicolumn{2}{|c|}{ Eclampsia } \\
\hline & \begin{tabular}{|c|} 
Magnesium \\
Sulphate \\
Group
\end{tabular} & $\begin{array}{c}\text { Control } \\
\text { Group }\end{array}$ & \begin{tabular}{|c|} 
Magnesium \\
Sulphate \\
Group
\end{tabular} & $\begin{array}{c}\text { Lytic } \\
\text { Cocktail } \\
\text { Group }\end{array}$ \\
\hline \begin{tabular}{|c|}
$\begin{array}{c}\text { Total No. of } \\
\text { Patients }\end{array}$ \\
\end{tabular} & 40 & 10 & 90 & 10 \\
\hline $\begin{array}{c}\text { No. of } \\
\text { Patients } \\
\text { (Occurrence/ } \\
\text { Recurrence } \\
\text { of Fit }\end{array}$ & 0 & 3 & 2 & 3 \\
\hline Percentage & 0 & $30 \%$ & $2.2 \%$ & 30 \\
\hline \multicolumn{5}{|c|}{$\begin{array}{l}\text { Table 4. Occurrence of Fits in Severe Preeclampsia and } \\
\text { recurrence of Fits in Eclampsia Cases }\end{array}$} \\
\hline
\end{tabular}




\begin{tabular}{|c|c|c|c|c|}
\hline \multirow{2}{*}{$\begin{array}{c}\text { Treatment } \\
\text { Delivery } \\
\text { Interval }\end{array}$} & $\begin{array}{c}\text { Severe Preeclampsia } \\
\text { Sagnesium } \\
\text { Sulphate } \\
\text { Group }\end{array}$ & $\begin{array}{c}\text { Control } \\
\text { Group }\end{array}$ & $\begin{array}{c}\text { Magnesium } \\
\text { Sulphate } \\
\text { Group }\end{array}$ & $\begin{array}{c}\text { Lytic } \\
\text { Cocktail } \\
\text { Group }\end{array}$ \\
\hline$<24 \mathrm{hrs}$. & $\begin{array}{c}27 \\
(67.5 \%)\end{array}$ & $\begin{array}{c}7 \\
(70 \%)\end{array}$ & $\begin{array}{c}64 \\
(83.11 \%)\end{array}$ & $\begin{array}{c}4 \\
(80 \%)\end{array}$ \\
\hline$>24 \mathrm{hrs}$. & $\begin{array}{c}13 \\
(32.5 \%)\end{array}$ & $\begin{array}{c}3 \\
(30 \%)\end{array}$ & $\begin{array}{c}13 \\
(16.9 \%)\end{array}$ & $\begin{array}{c}1 \\
(20 \%)\end{array}$ \\
\hline $\begin{array}{c}\text { Undelivered } \\
\text { (Died) }\end{array}$ & & & 2 & 1 \\
\hline Postpartum & \multicolumn{5}{|c|}{ Table 5. Treatment Delivery Interval in Four Treatment } \\
Groups
\end{tabular}

$\mathrm{p}=3.841$

\begin{tabular}{|c|c|c|c|c|}
\hline Mode of & \multicolumn{2}{|c|}{ Severe Preeclampsia } & \multicolumn{2}{c|}{ Eclampsia } \\
Delivery & $\begin{array}{c}\text { Magnesium } \\
\text { Sulphate } \\
\text { Group }\end{array}$ & $\begin{array}{c}\text { Control } \\
\text { Group }\end{array}$ & $\begin{array}{c}\text { Magnesium } \\
\text { Sulphate } \\
\text { Group }\end{array}$ & $\begin{array}{c}\text { Lytic } \\
\text { Cocktail } \\
\text { Group }\end{array}$ \\
\hline $\begin{array}{c}\text { Normal } \\
\text { Delivery }\end{array}$ & 24 & 6 & 44 & 3 \\
\hline Forceps & 2 & 1 & 11 & 1 \\
\hline Craniotomy & -- & -- & -- & -- \\
\hline $\begin{array}{c}\text { Postpartum } \\
\text { Eclampsia }\end{array}$ & -- & -- & 11 & 4 \\
\hline
\end{tabular}

Table 6. Mode of Delivery in Four Treatment Groups $\mathrm{p}=5.99$

\begin{tabular}{|c|c|c|c|c|}
\hline & \multicolumn{2}{|c|}{ Severe Preeclampsia } & \multicolumn{2}{|c|}{ Eclampsia } \\
\hline & $\begin{array}{l}\mathrm{MgSO}_{4} \\
\text { Group }\end{array}$ & $\begin{array}{l}\text { Control } \\
\text { Group }\end{array}$ & $\begin{array}{l}\mathrm{MgSO}_{4} \\
\text { Group }\end{array}$ & $\begin{array}{l}\text { Lytic } \\
\text { Cocktail } \\
\text { Group }\end{array}$ \\
\hline & $\begin{array}{c}\text { Total No. } \\
\text { of Cases } \\
(\%)\end{array}$ & $\begin{array}{c}\text { Total No. } \\
\text { of Cases } \\
(\%)\end{array}$ & $\begin{array}{c}\text { Total No. } \\
\text { of Cases } \\
\text { (\%) }\end{array}$ & \begin{tabular}{|c}
$\begin{array}{c}\text { Total No. } \\
\text { of Cases } \\
(\%)\end{array}$ \\
\end{tabular} \\
\hline Coma & 0 & 0 & $11(12.2 \%)$ & \begin{tabular}{l|l}
$5)$ & $4(40 \%)$
\end{tabular} \\
\hline Hyperpyrexia & 0 & 0 & $9(10 \%)$ & $1(10 \%)$ \\
\hline $\begin{array}{c}\text { Pulmonary } \\
\text { Oedema }\end{array}$ & 0 & 0 & $3(3.3 \%)$ & $1(10 \%)$ \\
\hline Oliguria & $4(10 \%)$ & $1(10 \%)$ & $8(8.8 \%)$ & $1(10 \%)$ \\
\hline UTI & $2(5 \%)$ & $1(10 \%)$ & $4(4.4 \%)$ & $1(10 \%)$ \\
\hline $\mathrm{PPH}$ & $1(2.5 \%)$ & $1(10 \%)$ & $4(4.4 \%)$ & $1(10 \%)$ \\
\hline $\begin{array}{l}\text { Puerperal } \\
\text { Psychosis }\end{array}$ & -- & -- & $1(1.1 \%)$ & $1(10 \%)$ \\
\hline \multicolumn{5}{|c|}{ Table 7. Maternal Pattern in Relation to Therapy } \\
\hline$=12.59$ & & & & \\
\hline & Severe Pree & eclampsia & Eclam & npsia \\
\hline & $\begin{array}{l}\mathrm{MgSO}_{4} \\
\text { Group }\end{array}$ & $\begin{array}{l}\text { Control } \\
\text { Group }\end{array}$ & $\begin{array}{l}\mathrm{MgSO}_{4} \\
\text { Group }\end{array}$ & $\begin{array}{c}\text { Lytic } \\
\text { Cocktail } \\
\text { Group }\end{array}$ \\
\hline & $\begin{array}{c}\begin{array}{c}\text { Total No. } \\
\text { of Cases } \\
(\%)\end{array} \\
\end{array}$ & \begin{tabular}{|c|}
$\begin{array}{c}\text { Total No. } \\
\text { of Cases } \\
(\%)\end{array}$ \\
\end{tabular} & $\begin{array}{c}\begin{array}{c}\text { Total No. } \\
\text { of Cases } \\
(\%)\end{array} \\
\end{array}$ & $\begin{array}{l}\text { Total No. of } \\
\text { Cases (\%) }\end{array}$ \\
\hline RTI & -- & -- & $8(8.8 \%)$ & $1(10 \%)$ \\
\hline Shock & -- & -- & $2(2.2 \%)$ & -- \\
\hline Bedsore & -- & -- & -- & -- \\
\hline $\begin{array}{l}\text { Abruptio } \\
\text { placentae }\end{array}$ & $4(10 \%)$ & $2(20 \%)$ & $6(6.6 \%)$ & $2(20 \%)$ \\
\hline $\begin{array}{c}\text { Pain at } \\
\text { injection } \\
\text { site }\end{array}$ & $1(2.5 \%)$ & 0 & $6(6.6 \%)$ & 0 \\
\hline DIC & 0 & 0 & $1(1.1 \%)$ & 0 \\
\hline CVA & 0 & 0 & $2(2.2 \%)$ & $1(10 \%)$ \\
\hline & & 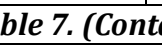 & & \\
\hline
\end{tabular}

\begin{tabular}{|c|c|c|c|c|}
\hline & \multicolumn{2}{|c|}{ Severe Preeclampsia } & \multicolumn{2}{c|}{ Eclampsia } \\
\cline { 2 - 5 } & $\begin{array}{c}\text { Magnesium } \\
\text { Sulphate } \\
\text { Group }\end{array}$ & $\begin{array}{c}\text { Control } \\
\text { Group }\end{array}$ & $\begin{array}{c}\text { Magnesium } \\
\text { Sulphate } \\
\text { Group }\end{array}$ & $\begin{array}{c}\text { Lytic } \\
\text { Cocktail } \\
\text { Group }\end{array}$ \\
\hline $\begin{array}{c}\text { No. of } \\
\text { Deaths }\end{array}$ & 0 & 0 & 6 & 2 \\
\hline $\begin{array}{c}\text { Maternal } \\
\text { Mortality }\end{array}$ & 0 & 0 & $6.6 \%$ & $20 \%$ \\
\hline
\end{tabular}

Table 8. Maternal Mortality of Four Treatment Groups $\mathrm{p}=0$

\begin{tabular}{|c|c|}
\hline Magnesium Sulphate Group & Lytic Cocktail Group \\
\hline Total No. of deaths- 6 (6.6\%) & Total No. of deaths- 2(20\%) \\
\hline Causes implicated & Causes implicated \\
\hline $\begin{array}{c}\text { Deep coma with } \\
\text { cardiorespiratory arrest- } 2\end{array}$ & $\begin{array}{c}\text { Deep coma with } \\
\text { cardiorespiratory arrest- } 1\end{array}$ \\
\hline Pulmonary oedema- 2 & \\
\hline Acute renal failure- 1 & Pulmonary oedema- 1 \\
\hline DIC and shock- 1 & Table 9. Causes of Maternal Death \\
\hline \multicolumn{2}{|c|}{}
\end{tabular}
$\mathrm{p}=0$

\begin{tabular}{|c|c|c|c|c|}
\hline & \multicolumn{2}{|c|}{ Severe Preeclampsia } & \multicolumn{2}{c|}{ Eclampsia } \\
\cline { 2 - 5 } & $\begin{array}{c}\text { Management } \\
\text { Sulphate } \\
\text { Group }\end{array}$ & $\begin{array}{c}\text { Control } \\
\text { Group }\end{array}$ & $\begin{array}{c}\text { Management } \\
\text { Sulphate } \\
\text { Group }\end{array}$ & $\begin{array}{c}\text { Lytic } \\
\text { Cocktail } \\
\text { Group }\end{array}$ \\
\hline $\begin{array}{c}\text { Total } \\
\text { Births }\end{array}$ & 40 & 10 & 88 & 9 \\
\hline \multicolumn{5}{|c|}{ Home Delivery } \\
\hline Alive & -- & -- & 6 & 1 \\
\hline Stillbirth & -- & -- & 4 & 1 \\
\hline $\begin{array}{c}\text { Neonatal } \\
\text { Death }\end{array}$ & -- & -- & 1 & \\
\hline $\begin{array}{c}\text { Table 10. Perinatal Outcome of all Births (Home or } \\
\text { Hospital) in the Four Management Groups }\end{array}$ \\
\hline
\end{tabular}

\begin{tabular}{|c|c|c|c|c|}
\hline & \multicolumn{2}{|c|}{$\begin{array}{c}\text { Severe } \\
\text { Preeclampsia }\end{array}$} & \multicolumn{2}{|c|}{ Eclampsia } \\
\hline & $\begin{array}{l}\mathrm{MgSO}_{4} \\
\text { Group }\end{array}$ & $\begin{array}{c}\text { Control } \\
\text { group }\end{array}$ & $\begin{array}{l}\mathrm{MgSO}_{4} \\
\text { Group }\end{array}$ & $\begin{array}{c}\text { Lytic } \\
\text { Cocktail } \\
\text { group }\end{array}$ \\
\hline \multicolumn{5}{|c|}{ Hospital Delivery } \\
\hline $\begin{array}{l}\text { Absent FHS on } \\
\text { admission }\end{array}$ & 1 & 0 & 10 & 1 \\
\hline $\begin{array}{l}\text { FHS present at } \\
\text { admission }\end{array}$ & 39 & 10 & 69 & 5 \\
\hline Stillbirth & 0 & 1 & 4 & 1 \\
\hline $\begin{array}{l}\text { Early neonatal } \\
\text { death }\end{array}$ & 0 & 0 & 3 & 1 \\
\hline $\begin{array}{c}\text { Perinatal death in } \\
\text { all patients }\end{array}$ & $2.5 \%$ & $10 \%$ & $24.4 \%$ & $50 \%$ \\
\hline $\begin{array}{c}\text { Neonatal mortality } \\
\text { in patients with } \\
\text { FHS present at } \\
\text { admission }\end{array}$ & 0 & $10 \%$ & $10.1 \%$ & $40 \%$ \\
\hline $\begin{array}{c}\text { Went home with } \\
\text { live baby }\end{array}$ & $97.5 \%$ & $90 \%$ & $75.6 \%$ & $50 \%$ \\
\hline \multicolumn{5}{|c|}{ Table 10. (Contd..) } \\
\hline
\end{tabular}




\begin{tabular}{|c|c|c|c|c|}
\hline & \multicolumn{2}{|c|}{$\begin{array}{c}\text { Severe } \\
\text { Preeclampsia }\end{array}$} & \multicolumn{2}{c|}{ Eclampsia } \\
\hline & $\begin{array}{c}\text { MgSO }_{4} \\
\text { Group }\end{array}$ & $\begin{array}{c}\text { Control } \\
\text { Group }\end{array}$ & $\begin{array}{c}\text { MgSO }_{4} \\
\text { Group }\end{array}$ & $\begin{array}{c}\text { Lytic } \\
\text { Cocktail } \\
\text { Group }\end{array}$ \\
\hline $\begin{array}{c}\text { Nil (No } \\
\text { complications) }\end{array}$ & $26(65 \%)$ & $5(50 \%)$ & $\begin{array}{c}46 \\
(51.1 \%)\end{array}$ & $1(10 \%)$ \\
\hline \multicolumn{4}{|c|}{ Birth Asphyxia } \\
\hline Apgar 4-6 & $8(20 \%)$ & $2(20 \%)$ & $\begin{array}{c}12 \\
(13.3 \%)\end{array}$ & $2(20 \%)$ \\
\hline Apgar 1-3 & 0 & $1(10 \%)$ & $2(2.2 \%)$ & $1(10 \%)$ \\
\hline $\begin{array}{c}\text { Meconium } \\
\text { Aspiration }\end{array}$ & $3(7.5 \%)$ & $1(10 \%)$ & $6(6.6 \%)$ & $1(10 \%)$ \\
\hline Septicaemia & 0 & 0 & $6(6.6 \%)$ & $1(10 \%)$ \\
\hline Hyperbilirubinaemia & 0 & 0 & $4(4.4 \%)$ & -- \\
\hline $\begin{array}{c}\text { Intracranial } \\
\text { Haemorrhage }\end{array}$ & 0 & 0 & -- & -- \\
\hline $\begin{array}{c}\text { Table 11. Neonatal Morbidity Pattern in Four Treatment } \\
\text { Groups }\end{array}$ \\
\hline
\end{tabular}

$\mathrm{p}=12.59$

\section{Statistical Analysis}

Qualitative variables were analysed using proportions. Quantitative variables were analysed using mean and standard deviation. Chi-square test was applied to note if there was any statistical significance between the treatment with magnesium sulphate and lytic cocktail in eclampsia and severe preeclampsia cases and a "P" value of less than .05 was considered statistically significant.

\section{RESULTS}

\section{Table III}

In Table No. III, diastolic blood pressure has been compared before starting the treatment and 24 hours after treatment in study and control group. P-value is 3.84 , that means it is not significant.

\section{Table IV}

In this table, comparison between occurrence of convulsion in severe PET and recurrence of fits in eclampsia in study group and control group has been done. P-value is 3.84 , that means it is not significant.

\section{Table V}

Treatment and delivery interval in four treatment groups has been shown. P-value is 3.84. This again means it is not significant.

\section{Table VI}

Shows mode of delivery in four treatment groups. P-value is 5.99 , that means treatment method does not have influence on mode of delivery.

\section{Table VIII}

Shows maternal mortality of four treatment groups. Here Pvalue is 0 , that means magnesium sulphate treatment reduces maternal mortality.

\section{Table IX}

Shows causes of maternal deaths. P-value is 0 , that means magnesium sulphate treatment reduces maternal mortality.
Table X

Shows perinatal outcome in different groups. P-value is 12.59 , that means it is insignificant.

\section{Table XI}

Shows neonatal morbidity pattern in four treatment groups. $\mathrm{P}$-value is 12.59 . This again means mode of treatment does not have significant role in neonatal morbidity.

\section{DISCUSSION}

Incidence of eclampsia in this study was $5.03 \%$, which is higher than previous studies. Incidence of imminent eclampsia was $3.45 \%$.

Maximum patients were below 20 years' age group, $82.7 \%$ cases were unbooked. Majority of patients in both the groups were from lower and lower-middle socioeconomic strata. $71.4 \%$ patients were primigravida. $77 \%$ patients had antepartum eclampsia, $15 \%$ patients had postpartum eclampsia and $8 \%$ patients had intrapartum eclampsia.

Diastolic blood pressure became less than or equal to 90 mmHg after treatment with magnesium sulphate in $60 \%$ cases of imminent eclampsia and $61.4 \%$ cases of eclampsia as compared to $40 \%$ in control group. There was no fit in imminent eclampsia patients treated with magnesium sulphate, whereas $30 \%$ of patients of control group had fits.

Maternal mortality was significantly lower in magnesium sulphate group than lytic cocktail group (6.6\% vs. $20 \%)$. Perinatal mortality was markedly better in magnesium sulphate group of imminent eclampsia patients as compared to control group ( $2.5 \%$ vs. $10 \%)$.

\section{CONCLUSION}

To conclude, it can be emphasised that magnesium sulphate is the drug of choice for the prophylactic (Duley 2003a) ${ }^{[9]}$ as well as therapeutic management of severe preeclampsia and eclampsia respectively (Duley 2003b; Duley 2003c).[10,11] It is very efficient in the prevention of fits in severe preeclampsia and reduction in recurrence of fits in eclampsia. $\mathrm{MgSO}_{4}$ is also efficient in reducing maternal morbidity and mortality. It also improved perinatal outcome.

\section{REFERENCES}

[1] Douglas KA, Redman CW. Eclampsia in the United Kingdom. BMJ 1994;309(6966):1395-400.

[2] Dinsdale HB. Does magnesium sulphate treat eclamptic seizures? Yes. Archives of Neurology 1988;45(12):1360-1.

[3] Pritchard JA. The use of the magnesium ion in the management of eclamtogenic toxemias. Surgery Gynaecology and Obstetrics 1955;100(2):131-40.

[4] Zuspan FP. Problems encountered in the treatment of pregnancy-induced hypertension. A point of view. American Journal of Obstetrics and Gynaecology 1978;131(6):591-7.

[5] Menon MK. The evolution of the treatment of eclampsia. Journal of Obstetrics \& Gynaecology of the British Commonwealth 1961;68:417-26.

[6] Euser AG, Cipolla MJ. Magnesium sulphate for the treatment of eclampsia: a brief review. Stroke 2009;40(4):1169-75. 
[7] Belfort MA, Moise KJ Jr. Effect of magnesium sulphate on maternal brain blood flow in pre-eclampsia: a randomized, placebo-controlled study. American Journal of Obstetrics \& Gynaecology 1992;167(3):661-6.

[8] Duckitt K, Harrington D. Risk factors for preeclampsia at antenatal booking: systematic review of controlled studies. BMJ 2005;330(7491):565.

[9] Duley L, Henderson-Smart DJ. Magnesium sulphate versus diazepam for eclampsia. Cochrane of Systematic Reviews 2003a, Issue 4. Art No: CD000127. DOI: 10.1002/14651858.CD000127.
[10] Duley L, Henderson-Smart DJ. Magnesium sulphate versus phenytion for eclampsia. Cochrane database of Systematic Reviews 2003b, Issue 4. Art No: CD000128. D0I: 10.1002/14651858.CD000128.

[11] Duley L, Gulmezoglu AM, Henderson-Smart DJ, et al. Magnesium sulphate and other anticonvulsants for women with pre-eclampsia. Cochrane Database of Systematic Reviews 2003c, Issue 2. Art No: CD000025. DOI: 10.1002/14651858.CD000025 